\title{
Comment on "Labia minora tourniquet syndrome in an adult resulting from erotic dancing"
}

\author{
Kathryn Chan $^{1} \oplus \cdot$ Lauren Cook-Chaimowitz ${ }^{1} \cdot$ Sameer Sharif ${ }^{1,2}$
}

Received: 17 September 2020 / Accepted: 24 September 2020 / Published online: 4 January 2021

(c) Canadian Association of Emergency Physicians (CAEP)/ Association Canadienne de Médecine d'Urgence (ACMU) 2020

\section{Dear Editor,}

The case report from Drs. Pelletier and Chagnon [1] describe a unique presentation of tourniquet syndrome. However, we have concerns with the language used to present the patient's narrative.

The case presentation includes several graphic details that are extraneous to the clinical context and unnecessarily sexualize the patient. For instance, the authors write that "[t]he patient acknowledged consuming several alcoholic drinks... participated in grinding - a type of erotic dance." Furthermore, they continue with their tone as follows, "[a] $t$ approximately 03:30, she was unable to remove her lace underwear". These specifiers distract from the potential learning in the case by, instead, bringing attention to the patient's clothing, motives, and substance use; features that do not change how we provide care.

A patient's history shapes our understanding of their case and influences our actions. Therefore, the language we use to communicate their story matters tremendously. A study of chart notes showed that stigmatizing language is associated with negative attitudes towards patients and even changes management when compared to charts written in a neutral tone [2]. Implicit bias is demonstrated in healthcare for numerous patient characteristics, including gender and substance use [3], both of which are relevant to this case.

It is especially important to be cautious of the language used in case reports, given the focus on one patient and the permanence of their personal experience in our medical literature. Objectifying language erodes the humanity that drives our therapeutic relationships. However, language

Kathryn Chan

Kathryn.chan@medportal.ca

1 Department of Medicine, Division of Emergency Medicine, McMaster University, Hamilton, ON, Canada

2 Department of Medicine, Division of Critical Care, McMaster University, Hamilton, ON, Canada can also present an opportunity to build partnership through mutually agreed terminology, for instance, person-first language ('person with schizophrenia' vs. 'schizophrenic') and nonjudgmental terms ('noncompliant' vs. 'declined,' 'getting clean' vs. 'in recovery') [4].

Narrative medicine is a growing field that underscores how building a story is a collaborative act between physician and patient [5]. When done intentionally, it can create rapport and convey empathy. Conversely, misrepresentation of a patient's account threatens this alliance. Our words hold immense power.

Every day, in emergency departments, patients share their most intimate and personal fears, and trust us to use that information appropriately. It is our collective responsibility, including the editors of journals, to continuously improve our profession by sharing their stories with dignity and respect.

\section{References}

1. Pelletier A, Chagnon N. Labia minora tourniquet syndrome in an adult resulting from erotic dancing. CJEM 2020;22(5):721-2.

2. Goddu AP, O'Conor KJ, Lanzkron S, Saheed MO, Saha S, Peek $\mathrm{ME}$, et al. Do words matter? Stigmatizing language and the transmission of bias in the medical record. J Gen Intern Med. 2018;33(5):685-91.

3. Fitzgerald C, Hurst S. Implicit bias in healthcare professionals: a systematic review. BMC Med Ethics. 2017;18(19).

4. Carroll SM. Respecting and empowering vulnerable populations: contemporary terminology. J Nurse Pract. 2019;15(3):228-31.

5. Charon R. Narrative medicine a model for empathy, reflection, profession, and trust. JAMA. 2001;286(15):1897-902. 